\title{
Low sensitivity of ParaHIT-f rapid malaria test among patients with fever in rural health centers, Northern Tanzania
}

\author{
Eliningaya J. Kweka ${ }^{1}$, Asanterabi Lowassa ${ }^{2}$, Shandala Msangi ${ }^{1}$, Epiphania E. Kimaro ${ }^{1}$, Ester E. \\ Lyatuu $^{1}$, Beda J. Mwang'onde ${ }^{1}$, Aneth M. Mahande ${ }^{3}$, Humphrey D. Mazigo ${ }^{4}$ \\ ${ }^{1}$ Tropical Pesticides Research Institute, Division of Livestock and Human Disease Vectors Control, PO Box 3024, \\ Arusha, Tanzania \\ ${ }^{2}$ Tanzania Wildlife Research Institute, PO Box 661, Arusha, Tanzania \\ ${ }^{3}$ Tropical Pesticides Research Institute, Mabogini field station, Moshi, Tanzania \\ ${ }^{4}$ Department of Medical Parasitology and Entomology, Weill-Bugando University College of Health Sciences, PO \\ Box 1464, Mwanza, Tanzania
}

\begin{abstract}
Introduction: Several rapid diagnostic tools for malaria are currently available in local markets. However, diagnostic accuracy varies widely. The present study was conducted to evaluate a cheaply and easily available rapid diagnostic malaria test (ParaHIT- $f$ ) in rural Tanzania.

Methodology: Participants presenting with fever at health centers in the Kilimanjaro and Manyara regions were eligible. Parasitological thin and thick smears were examined from finger-prick blood samples and compared to ParaHIT- $f$ test results. Sensitivity, specificity and predictive values were calculated using microscopic parasitological examination as the gold standard.

Results: In total, 236/743 (31.8\%) individuals had a positive malaria microscopy, and 25/715 (3.4\%) were positive in the rapid diagnostic test. The sensitivity of ParaHIT- $f$ was $10.7 \%$ (95\% CI, 6.7-14.7) and specificity was $100 \%$ (95\% CI, 97.4-102), with positive and negative predictive values (PPV and NPV) of $100 \%$ (95\% CI, 99.1-100.2) and 70.9\% (95\% CI, 66.9-74.9) respectively. Sensitivity of ParaHIT- $f$ increased with increasing $P$. falciparum density $(P>0.003)$ from $5.8 \%(95 \% \mathrm{CI}, 0-12.9)$ at $<100$ parasites $/ \mu 1$ to $20.5 \%(95 \% \mathrm{CI}, 13.5-27)$ at $\geq 100$ parasites $/ \mu 1$.

Conclusions: Sensitivity of the ParaHIT- $f$ rapid test was very low in this setting, therefore concomitant use of rapid diagnostic tests and microscopy is recommended. In the case of positive test results, confirmation by parasitological techniques is not necessary. Further monitoring of ParaHIT- $f$ in various epidemiological settings in Tanzania is warranted.
\end{abstract}

Key words: RDT; Plasmodium falciparum; malaria; Tanzania

J Infect Dev Ctries 2011; 5(3):204-208.

(Received, 30 June 2010 -- Accepted, 07 October 2010)

Copyright () 2011 Kweka et al. This is an open-access article distributed under the Creative Commons Attribution License, which permits unrestricted use, distribution, and reproduction in any medium, provided the original work is properly cited.

\section{Introduction}

Among the most pronounced problems in controlling the morbidity and mortality caused by malaria is the limited access to effective diagnosis in endemic areas. In these areas, malaria is commonly over-diagnosed [1-3]. In Tanzania, up to $90 \%$ of cases diagnosed as malaria in outpatient departments had no positive blood slide for malaria parasites $[3,4]$. Some causes of over-diagnosis include clinical signs and symptoms that overlap with other infections [5]. As a consequence, patients presenting with fever are often presumptively diagnosed as malaria cases, without any parasitological confirmation [6].

For effective malaria case management, timely and early diagnosis and prompt treatment (EDPT) with efficacious drugs is required. However, in most malarial endemic areas, diagnosis still relies on the use of microscopy. The technique is regarded as very old, labor intensive and furthermore, it requires significant skills, electricity and time, which cause therapeutic delays $[7,8]$.

To overcome the limitations of microscopy, a number of rapid diagnostic tests (RDTs) for malaria have been developed. One of these is ParaHIT- $f$ which is currently marketed and used for malaria diagnosis in various regions in Tanzania and other African countries [9]. The test has been evaluated in various epidemiological settings in malarial endemic areas and the results on its sensitivity, specificity, positive predictive values (PPV), and negative 
Table 1. Demographic characteristics of 745 patients from Mabogini and Magugu, northern Tanzania.

\begin{tabular}{lcc}
\hline Characteristics & N & \% \\
\hline Gender & 342 & 45.9 \\
Male & 403 & 54.1 \\
Female & & \\
Age groups (years) & 284 & 38.1 \\
$0-5$ & 148 & 19.9 \\
$6-20$ & 160 & 21.6 \\
$21-35$ & 86 & 11.5 \\
$36-50$ & 67 & 8.9 \\
$51+$ & 745 & 100.0 \\
\hline Total &
\end{tabular}

predictive values (NPV) vary significantly [10,11]. For example, several studies from India and Tanzania have shown a high sensitivity and specificity of the ParaHIT- $f$ rapid diagnostic test for diagnosis of Plasmodium falciparum malaria [9,12]. However, we have previously shown that, in a rural Tanzanian setting, sensitivity was low [10]. Thus monitoring the performance of ParaHIT- $f$, which is the most commonly used and inexpensive test available for malaria diagnosis in Tanzanian markets, remains important for accurate diagnosis and timely treatment of the disease. It also reduces both the misuse of antimalarials, artemisinin-based combination therapy (ACT) and mortality, especially in young children.

The present study was therefore conducted to confirm previous findings and to evaluate the diagnostic accuracy of ParaHIT- $f$ in two rural areas located in low to moderate malaria transmission zones and epidemic prone areas. The results of this study will help decision makers to decide whether to use this RDT for routine malaria diagnosis in health facilities.

\section{Methodology}

\section{Study areas}

This cross-sectional study was conducted from May to June 2008 in two communities with similar agro-ecosystems. Lower Moshi in the Kilimanjaro region is an area located at the base of Mount Kilimanjaro ( $\left.3^{\circ} 21^{\prime} \mathrm{S}, 37^{\circ} 21^{\prime} \mathrm{E}\right)$ at an altitude of 800 meters above sea level. The area receives between $900 \mathrm{~mm}$ and $1200 \mathrm{~mm}$ of rainfall per year and is located in a holoendemic malaria transmission zone [13]. The other area, Magugu, is located in the Manyara region within the northern Great Rift Valley area $\left(3^{\circ} 53^{\prime} \mathrm{S}, 35^{\circ} 42^{\prime} \mathrm{E}\right)$ at an altitude of 969 meters above sea level. The area receives about $1500 \mathrm{~mm}$ of rainfall per year. Magugu ward is located in an area with moderate malaria transmission and is prone to malaria epidemics [14]. In these two areas, malaria transmission is more intense between April and midJune to early July, and more malaria cases are reported in the same period $[13,14]$. This study was conducted in June 2008 when it was transmission season. The communities within these two areas are involved in rice farming and livestock keeping.

\section{Study population, recruitment and sampling}

The study recruited patients of all age groups reporting at these two health centers with either fever (temperature $>37.5^{\circ} \mathrm{C}$ ), history of fever in the preceding two or three days, or symptoms suggestive of malaria. Patients were recruited serially until the sample size of 745 patients was reached (Table 1).

All patients were attended by clinicians and after consultation were directed to seek laboratory investigation. For diagnosis of malaria, a finger-prick blood sample was collected from each consenting patient. This sample was used to prepare thin and thick smears, which were dried at room temperature and stained with Giemsa following World Health Organization (WHO) standards [15]. All slides obtained were examined by two independent, experienced microscopists blinded to the clinical status of the patients and the results of RDTs. Parasitemia was determined from the thick films by counting the number of parasites against 200 leucocytes and assuming that each subject had 8000 leucocytes $/ \mu$ l. The thin smears served to identify malaria parasite species [15]. A slide was concluded 
Table 2. Overall sensitivity, specificity, positive and negative predictive values (PPV, NPV) of ParaHIT$f$, and stratified results according to parasite density.

\begin{tabular}{|c|c|c|c|c|}
\hline $\begin{array}{c}\text { Parasitemia } \\
\text { (parasites/ } \mu \mathrm{l} \text { ) }\end{array}$ & $\begin{array}{l}\text { Sensitivity } \\
(95 \% \text { CI })\end{array}$ & $\begin{array}{l}\text { Specificity } \\
(95 \% \text { CI })\end{array}$ & $\begin{array}{c}\text { PPV } \\
(95 \% \mathrm{CI})\end{array}$ & $\begin{array}{c}\text { NPV } \\
(95 \% \mathrm{CI})\end{array}$ \\
\hline$<100$ & $5.8 \%(0-12.9)$ & $100 \%$ & $100 \%$ & $77.6 \%(74.6-81.1)$ \\
\hline$\geq 100$ & $20.5 \%(13.5-27)$ & $100 \%$ & $100 \%$ & $1.6 \%(0-8.6)$ \\
\hline Overall & $10.7 \%(6.7-14.7)$ & $100 \%$ & $100 \%$ & $70.9 \%(66.9-74.9)$ \\
\hline
\end{tabular}

to be negative when no parasites were observed after counting 200 white blood cells in different fields. All discordant slides and $15 \%$ of randomly selected slides were re-examined by a third expert for quality control.

The ParaHIT- $f$ test (Span diagnostic Ltd, Udhna, Surat-394210, India) was performed per the manufacturer's instructions [16]. This test detects $P$. falciparum in whole blood, based upon an antigen capture assay detecting the $P$. falciparum-specific histidine-rich protein II. Thus, it does not cross-react with other malaria parasites. The test is based upon immunochromatographic test strips and does not require any specialized skills. Briefly, about $5 \mu \mathrm{L}$ of blood was applied on the test kit and $200 \mu \mathrm{L}$ of the reaction buffer was added. The standard reading time was between 15 and 30 minutes.

Patients diagnosed with malaria by ParaHIT- $f$ and microscopy were treated according to national guidelines for the treatment of malaria using combination therapy (Artemether and Lumefantrine).

\section{Ethical considerations}

This study was approved by the institutional Ethical Review Committee of the Tropical Pesticides Research Institute, Arusha, Tanzania. Written consent was obtained from patients and in the case of children from their parents or guardians.

\section{Data analysis and management}

Data were entered and verified using MS-Access, and analyzed using STATA software (Stata Corp, College Station, TX, USA). Specificity, sensitivity and predictive values of ParaHIT- $f$ and their respective $95 \%$ confidence intervals were estimated using microscopy as the reference diagnosis (gold standard) [14]. ParaHIT- $f$ was also tested across different parasitemia levels. Parasitemia was categorized as either low ( $<100$ parasites $/ \mu$ l) or high ( $\geq 100$ parasites $/ \mu 1)$.

\section{Results}

A total of 745 patients were recruited in this study, $403(54.1 \%)$ of whom were females. The mean age was $20.1 \pm 20.3$ and the median age was 14 years (range 12 weeks to 84 years).

A total of $236(31.8 \%, 95 \%$ CI, 25.8-37.8) out of 743 patients had positive thick films for $P$. falciparum (two patients were omitted during analysis due to missing slide results). The asexual parasite density ranged from 40 to 14,680 parasites $/ \mu 1$. Discrepancies were found between the results of the first and second microscopist in 20/743 slides; hence the level of agreement between readers was $97.3 \%$.

The ParaHIT- $f$ rapid diagnostic test (RDT) detected malaria infections in $25(3.5 \%, 95 \% \mathrm{CI}, 0-$ 10.6) out of 715 patients (30 patients were omitted due to missing results as they refused consent for the rapid diagnostic test).

Sensitivity of the RDT was $<11 \%$, but specificity was high with no false positive results. The overall sensitivity, specificity, positive predictive (PPV) and negative predictive values (NPV) of ParaHIT- $f$ using microscopy as the gold standard are presented in Table 2. Sensitivity increased from $6 \%$ to $21 \%$ with increasing parasite density $(P<0.003$; Table 2).

\section{Discussion}

For effective malaria case management in endemic areas, timely and accurate diagnosis is required to reduce mortality, especially for individuals with low immunity such as young children [15]. The use of a good RDT, therefore, can make possible early and accurate diagnosis, which will lead to appropriate and timely treatment [17]. However, the poor sensitivity of most of the inexpensive RDTs that are available, such as ParaHIT- $f$, may hinder their reliability or utilization for malaria diagnosis in areas where microscopy is not available.

In the present study, the overall sensitivity of ParaHIT- $f$ was low (10.7\%). A similarly low 
sensitivity of the same test (9.6\%) has been recorded in the Philippines, and a sensitivity of $29.8 \%$ to $P$. falciparum was reported from a holoendemic area of Tanzania in previous studies $[10,17]$. The observed results were inconsistent with those from similar studies in India and Tanzania, which have reported high sensitivity of the test raging from $70 \%-97 \%$ in different epidemiological settings $[9,10,18,19]$. It appears that the sensitivity of this test varies widely from one ecological setting to another $[10,11,16,17]$. Importantly, the poor sensitivity of the locally available ParaHIT- $f$ in the market may have serious implications on the management of malaria cases and may lead to delays in the proper administration of anti-malaria drugs to patients.

On the other hand, the value of the ParaHIT- $f$ specificity observed in the present study is consistent with the results of other studies conducted elsewhere $[10,11,16,17]$. The specificity appears to be consistent from various areas ranging from $75 \%$ to $100 \%[10,11,16,17]$. The high specificity of the test is important for identification and differentiation of malaria parasites species; thus a positive test result would not need microscopic confirmation.

In the present study, ParaHIT- $f$ sensitivity increased considerably with parasite density, from $6 \%$ to $21 \%$. The WHO recommends a sensitivity of $95 \%$ at a parasitemia of 100 parasites/ $\mu 1$ as a target for RDT performance [20]; however, this investigation found unexplained poor sensitivity of the test at different levels of parasitemia as compared to WHO recommendations [20] and the results of other reports $[10,17]$. Similar observations to ours have been reported from elsewhere $[17,21,22]$.

The poor performance of ParaHIT- $f$ may be attributed to the end user as a result of poor storage, transportation and other possible causes described elsewhere [17]. A particular concern here is the poor sensitivity of ParaHIT- $f$ in detecting patients with low parasitemia; this has serious implications on malaria case management, especially in areas where treatment decisions are based on the RDT results. The use of ParaHIT $-f$ in areas with low to moderate malaria transmission is of limited use because in these areas most of the patients may present with low parasitemia which cannot be detected by the test. This may have a serious impact on patients; for instance, children may develop cerebral malaria as a result of delays in starting treatment.

This study has several limitations which could have affected the findings; for example, a fixed number of RDTs was used (745). Furthermore, because of financial constraints, the study was completed in a short period of time and therefore a very short time was taken to assess the relevant population. Future studies should be conducted to assess the stability and reliability of these RDTs in different weather conditions and storage facilities.

\section{Conclusion}

Our data showed that in a setting with low to moderate transmission in rural Tanzania, the sensitivity of ParaHIT- $f$ was low. We recommend the test to be used in conjunction with microscopy to improve diagnosis of malaria in these settings where mixed infections might be the source of malaria rather than $P$. falciparum alone.

\section{Acknowledgements}

We sincerely acknowledge all the patients for their voluntary participation in the study. We also thank the health workers for their positive support during data collection. This study was supported by the Tropical Pesticides Research Institute Internal Research funds.

\section{References}

1. Amexo M, Tolhurst R, Barnish G, Bates I (2004) Malaria misdiagnosis: effects on the poor and vulnerable. Lancet 364: 1896-1898.

2. Barat L, Chipipa J, Kolczak M, Sukwa T (1999) Does the availability of blood slide microscopy for malaria at health centers improve the management of persons with fever in Zambia? Am J Trop Med Hyg 60: 1024-1030.

3. Reyburn H, Mbatia R, Drakeley C, Carneiro I, Mwakasungula E, Mwerinde O, Saganda K, Shao J, Kitua A, Olomi R, Greenwood BM, Whitty CJ (2004) Overdiagnosis of malaria in patients with severe febrile illness in Tanzania: a prospective study. British Med J 329: 1212.

4. Reyburn H, Mbakilwa H, Mwangi R, Mwerinde O, Olomi R, Drakeley C, Whitty CJ (2007) Rapid diagnostic tests compared with malaria microscopy for guiding outpatient treatment of febrile illness in Tanzania: randomised trial. British Med J 334: 403.

5. Chandramohan D, Carneiro I, Kavishwar A, Brugha R, Desai V, Greenwood B (2001) A clinical algorithm for the diagnosis of malaria: results of an evaluation in an area of low endemicity. Trop Med Int Health 6: 505-510.

6. D'Acremont V, Lengeler C, Mshinda H, Mtasiwa D, Tanner M, Genton B (2009) Time to move from presumptive malaria treatment to laboratory-confirmed diagnosis and treatment in African children with fever. PLoS Med 6: e252.

7. Marsh K, English M, Peshu N, Crawley J, Snow R (1996) Clinical algorithm for malaria in Africa. Lancet 347: 13271328.

8. White NJ (1996) The treatment of malaria. N Engl J Med 335: 800-806.

9. McMorrow ML, Masanja MI, Kahigwa E, Abdulla SM, Kachur SP (2010) Quality assurance of rapid diagnostic tests for malaria in routine patient care in rural Tanzania. Am J Trop Med Hyg 82: 151-155. 
10. Kamugisha E, Mazigo HD, Manyama M, Rambau P, Mirambo M, Kataraihya JB, Mshana SE (2009) Low sensitivity but high specificity of ParaHIT-f in diagnosing malaria among children attending outpatient department in Butimba District Hospital, Mwanza, Tanzania. Tanz J Health Res 1: 97-99.

11. Singh N, Mishra AK, Shukla MM, Chand SK, Bharti PK (2005) Diagnostic and prognostic utility of an inexpensive rapid on site malaria diagnostic test (ParaHIT f) among ethnic tribal population in areas of high, low and no transmission in central India. BMC Infect Dis 5: 50.

12. Singh N and Saxena A (2005) Usefulness of a rapid on-site Plasmodium falciparum diagnosis (Paracheck PF) in forest migrants and among the indigenous population at the site of their occupational activities in central India. Am J Trop Med Hyg 72: 26-29.

13. Ijumba JN, Mosha FW, Lindsay SW (2002) Malaria transmission risk variations derived from different agricultural practices in an irrigated area of northern Tanzania. Med Vet Entomol 16: 28-38.

14. Mboera LE and Kitua AY (2001) Malaria epidemics in Tanzania: An overview. Afr J Health Sci 8: 17-23.

15. World Health Organization (2000) Malaria diagnostics, New Perspectives. WHO/MAL, 1091: 4-29.

16. Guthmann JP, Ruiz A, Priotto G, Kiguli J, Bonte L, Legros D (2002) Validity, reliability and ease of use in the field of five rapid tests for the diagnosis of Plasmodium falciparum malaria in Uganda. Trans R Soc Trop Med Hyg 96: 254257.

17. Belizario VY, Pasay CJ, Bersabe MJ, de Leon WU, Guerrero DM, Bugaoisan VM (2005) Field evaluation of malaria rapid diagnostic tests for the diagnosis of $\mathrm{P}$. falciparum and non-P. falciparum infections. Southeast Asian J Trop Med Public Health 36: 552-561.

18. Devi G, Indumathi VA, Sridharan D, Srinivas BP, Sandhya BM (2002) Evaluation of ParaHITf strip test for diagnosis of malarial infection. Indian J Med Sci 56: 489-494.

19. Singh N, Saxena A, Valecha N (2000) Field evaluation of the ICT malaria P.f/P.v immunochromatographic test for diagnosis of Plasmodium falciparum and $P$. vivax infection in forest villages of Chhindwara, central India. Trop Med Int Health 5: 765-770.

20. World Health Organization (2003) Informal consultation on field trials and quality assurance of malaria rapid diagnostic tests. WHO-WPR.

21. Huong NM, Davis TM, Hewitt S, Huong NV, Uyen TT, Nhan DH, Cong le D (2002) Comparison of three antigen detection methods for diagnosis and therapeutic monitoring of malaria: a field study from southern Vietnam. Trop Med Int Health 7: 304-308.

22. Mason DP, Kawamoto F, Lin K, Laoboonchai A, Wongsrichanalai C (2002) A comparison of two rapid field immunochromatographic tests to expert microscopy in the diagnosis of malaria. Acta Trop 82: 51-59.

\section{Corresponding author}

Eliningaya John Kweka

Tropical Pesticides Research Institute

United Public of Tanzania

Telephone: +255787745555

Fax: +255-27-2508042

pat.kweka@gmail.com

Conflict of interests: No conflict of interests is declared. 